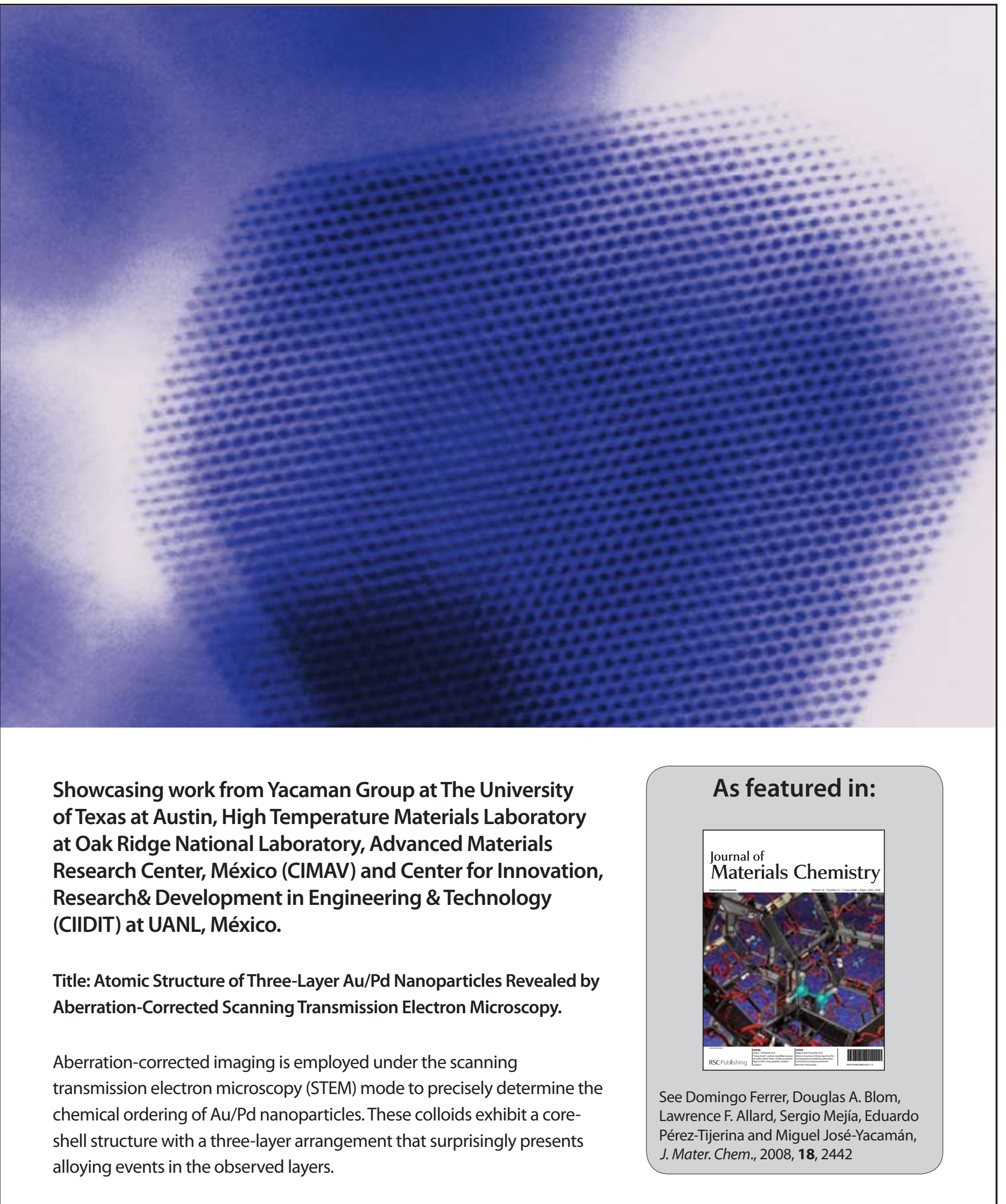




\title{
Atomic structure of three-layer Au/Pd nanoparticles revealed by aberration-corrected scanning transmission electron microscopy
}

\author{
Domingo Ferrer, ${ }^{a}$ Douglas A. Blom, ${ }^{b}$ Lawrence F. Allard, ${ }^{b}$ Sergio Mejía, ${ }^{c}$ Eduardo Pérez-Tijerina ${ }^{c d}$ \\ and Miguel José-Yacamán $† * a$
}

\author{
Received 23rd January 2008, Accepted 21st February 2008 \\ First published as an Advance Article on the web 19th March 2008 \\ DOI: $10.1039 / \mathrm{b801320a}$
}

\begin{abstract}
The study of nanomaterials can be greatly improved with the use of aberration-corrected transmission electron microscopy (TEM), which provides image resolutions at the level of $1 \AA$ and lower.

Sub-Ångström image resolution can yield a new level of understanding of the behavior of matter at the nanoscale. For example, bimetallic nanoparticles are extremely important in catalysis applications; the addition of a second metal in many cases produces much-improved catalysts. In this paper, we study the structure and morphology of $\mathrm{Au} / \mathrm{Pd}$ bimetallic particles using primarily the high-angle annular dark-field (HAADF) imaging mode in an aberration-corrected STEM/TEM. It is well established that, when recorded under appropriate illumination and collection geometries, incoherent HAADF-STEM images are compositionally sensitive and provide direct information on atomic positions. We matched the experimental intensities of atomic columns with theoretical models of three-layer $\mathrm{Au} / \mathrm{Pd}$ nanoparticles, in different orientations. Our findings indicate that the surface layer of the nanoparticle contains kinks, terraces and steps at the nanoscale. The effect of adding a second metal induces the formation of such defects, which might very likely promote the well-known improved catalytic activity of this system.
\end{abstract}

\section{Introduction}

Since the development of practical aberration correctors for electron microscopy, ${ }^{1}$ a new possibility for understanding matter at the nanolevel has been opened. In particular, aberrationcorrected scanning transmission electron microscopy (STEM) offers the possibility of studying atomic structures at a resolution of $<1 \AA,^{2-4}$ using primarily the high-angle annular dark-field (HAADF) imaging mode of an instrument with an aberration corrector fitted on the incident probe side. The incoherent nature of HAADF images, recorded with carefully controlled illumination and collection geometries, makes them free of dynamical interference effects (particularly for the case of crystalline particles of small dimensions) and therefore more straightforward to interpret than conventional high-resolution transmission electron microscopy (HRTEM) images. ${ }^{5-8}$

The structure of nanoparticles is of great interest in many applications of nanotechnology such as catalysis, plasmonics, sensing and others. Using two metals, we can achieve a broader range of properties; for instance, a core-shell structure will

${ }^{a}$ Chemical Engineering Departmental, The University of Texas at Austin, Austin, TX, 78712,USA. E-mail: yacaman@che.utexas.edu

${ }^{b}$ Materials Science and Technology Division, Oak Ridge National Laboratory, Oak Ridge, TN, 37831, USA

${ }^{c}$ Center for Innovation, Research \& Development in Engineering \& Technology (CIIDIT), Universidad Autónoma de Nuevo León, Monterrey, Nuevo León, 64440, México

${ }^{d}$ Advanced Materials Research Center, México (CIMAV), Chihuahua, 31109, México

$\dagger$ Currently working at: Department of Physics and Astronomy, The University of Texas at San Antonio, San Antonio, Texas, 78249, USA. E-mail:miguel.yacaman@utsa.edu scatter light in a different way than will an alloy particle. ${ }^{9} \mathrm{We}$ can in principle tune the properties by changing the way the metals combine. The case of $\mathrm{Au} / \mathrm{Pd}$ is particularly interesting. In the bulk, these two metals are fully soluble at all compositions. However, the $\mathrm{Au} / \mathrm{Pd}$ nanoparticle structure strongly depends on the preparation method. For instance, core-shell structures have been reported by several authors ${ }^{10-12}$ when chemical synthesis methods are used. On the other hand, $\mathrm{Au} / \mathrm{Pd}$ particles grown by sputtering show an alloy structure with an icosahedral structure. ${ }^{13}$ It is also possible to produce a three-layer structure, as shown by Marzan et al. ${ }^{14,15}$ In this work we describe a novel three-layer structure in $\mathrm{Au} / \mathrm{Pd}$ nanoparticles, and demonstrate that by combining aberrationcorrected imaging with conventional techniques such as theoretical simulation and single particle diffraction, we can obtain significant new information about nanoscale colloids.

\section{Experimental}

\section{Bimetallic nanoparticle synthesis}

The colloidal dispersions of $\mathrm{Au} / \mathrm{Pd}$ bimetallic clusters, stabilized by a polymer, were fabricated as previously described ${ }^{16}$ by successive alcoholic reduction of their constituent metal ions. Solutions of $0.1 \mathrm{M}$ tetrachloroauric acid $\left(\mathrm{HAuCl}_{4}\right)$ and $0.05 \mathrm{M}$ palladium chloride $\left(\mathrm{PdCl}_{2}\right)$ were prepared by dissolving the corresponding crystalline material in deionized water. The protective agent $\operatorname{poly}(N$-vinyl-2-pyrrolidone) (PVP, $0.4 \mathrm{~g}, 3.6 \mathrm{mmol}$ of monomeric units) and $\mathrm{PdCl}_{2}$ solution ( $\left.2 \mathrm{~mL}, 0.1 \mathrm{mmol}\right)$ were then mixed in ethylene glycol. This solution was stirred and heated to reflux at $140{ }^{\circ} \mathrm{C}$ in air. The reduction of the Pd precursor was indicated by a sudden change in the color of the solution from yellow to 
brown. Following reduction of the $\mathrm{Pd}$, the aqueous solution of $\mathrm{HAuCl}_{4}(1 \mathrm{~mL}, 0.1 \mathrm{mmol})$ was added and subsequently reduced. This mixture was finally refluxed again at $140{ }^{\circ} \mathrm{C}$ for $3 \mathrm{~h}$ in air. The total amount of both metals was always kept as $0.2 \mathrm{mmol}$ in $50 \mathrm{~mL}$ of the mixed solution. Nanoparticles with different compositions of gold and palladium (namely AuPd, $\mathrm{Au}_{0.75} \mathrm{Pd}_{0.25}$, and $\mathrm{Au}_{0.25} \mathrm{Pd}_{0.75}$ ), and monometallic nanoparticles of $\mathrm{Au}$ and $\mathrm{Pd}$, were fabricated by the above method.

\section{Transmission electron microscopy characterization}

Nanodiffraction patterns were acquired using a JEOL 2010F $(200 \mathrm{kV})$ transmission electron microscope equipped with a field-emission gun and an ultra-high-resolution pole piece with STEM attachment. Aberration-corrected microscopy was performed with a JEOL 2200FS STEM with a hexapole corrector (CEOS $\mathrm{GmbH})$ for the electron probe. The geometric aberrations of the probe-forming system were controlled to allow a beam convergence of $26.5 \mathrm{mrad}$ half-angle to be selected. The HAADF images were acquired from 100 to $170 \mathrm{mrad}$ scattering semi-angle, easily satisfying the requirement for the detector to eliminate contributions from unscattered or low-angle scattered beams. The theoretical models of the nanoparticles' structure were obtained using the Materials Studio software (Accelrys Inc.). In all cases the structure was relaxed to obtain the minimum energy configuration. The resultant atomic coordinates were fed into image simulation programs. We employed the SimulaTem ${ }^{17}$ software, modified for STEM, and the software WIN-HAADF (HREM Research Inc.). ${ }^{18}$

\section{Results and discussion}

The fabricated particles presented a three-layer structure, as has been previously reported. ${ }^{16}$ They exhibit a Pd-rich core, with the first shell highly enriched in Au, and a Pd-rich outer shell. A typical HAADF image of the three-layer $\mathrm{Au} / \mathrm{Pd}$ particles is shown in Fig. 1a. The inset indicates a sketch of element-rich locations in the layers. By tuning the ratio of $\mathrm{Au}$ and $\mathrm{Pd}$, the thickness of the layers systematically changed. A higher content of gold increased the diameter of the central Au-rich layer at the expense of the Pd-rich core and external thickness, and vice versa. The threelayer structure observed in these nanoparticles was found to be dependent on the diameter. ${ }^{16}$ It was observed that $25 \%$ of the nanoparticles had a diameter of $5.5 \pm 1.3 \mathrm{~nm}$, and only displayed two clear layers. The remaining $75 \%$ of the nanoparticles showed a size of $12.7 \pm 0.8 \mathrm{~nm}$, with three layers being evident.

The intensity profile through a typical $\mathrm{Au} / \mathrm{Pd}$ nanoparticle is displayed in Fig. 1b, and depends on the atomic number and column thickness. The crystallographic structure of these particles was directly determined by means of electron microdiffraction techniques from isolated nanostructures. A beam of 2-5 nm was precisely positioned on a particle and the diffraction pattern was obtained. Fig. 1c shows the microdiffraction patterns from two particles, consistent with $<100>$ and $<110>$ orientations of an FCC structure. The lattice parameter was measured by using the standard non-linear form of the Bragg Law, ${ }^{19}$ and a value of $3.97 \AA$ was obtained.

An ultra-high-resolution HAADF image of $\mathrm{Au} / \mathrm{Pd}$ nanoparticles is shown in Fig. 2a. The atomic columns are clearly

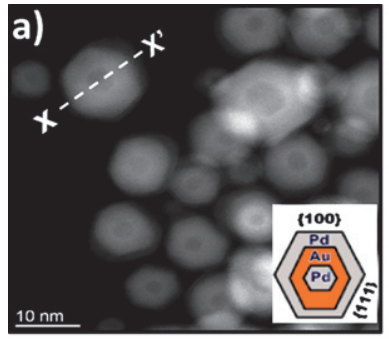

c) $\langle 100\rangle$

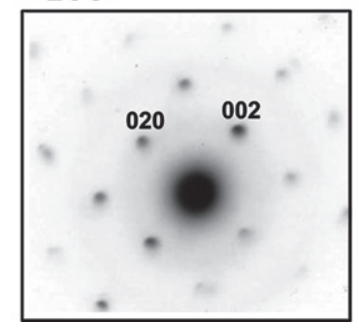

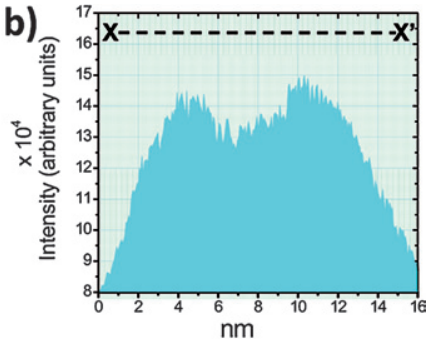

$\mathrm{nm}$

$<110\rangle$

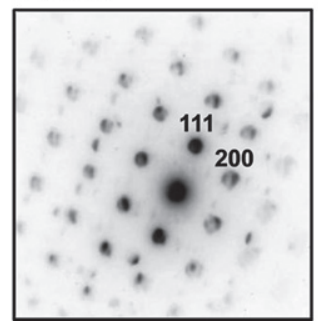

Fig. 1 (a) Image of $\mathrm{Au} / \mathrm{Pd}$ nanoparticles with cuboctahedral shape. The contrast is due to a core-shell structure consisting of three layers as sketched in the inset. Projected facets of the cuboctahedral particles can be observed. (b) The experimental intensity profile of a typical HAADF-STEM image shows a lower magnitude on the central portion on the particle (indicated as $\mathrm{x}-\mathrm{x}^{\prime}$ ) due to the fact that $Z_{\mathrm{Pd}}<Z_{\mathrm{Au}}$. (c) Nanodiffraction patterns of an individual $\mathrm{Au} / \mathrm{Pd}$ nanoparticle showing a single-crystalline structure. Two orientations, $<100>$ and $<110>$, can be observed. Note that extra spots due to twins are not observed.
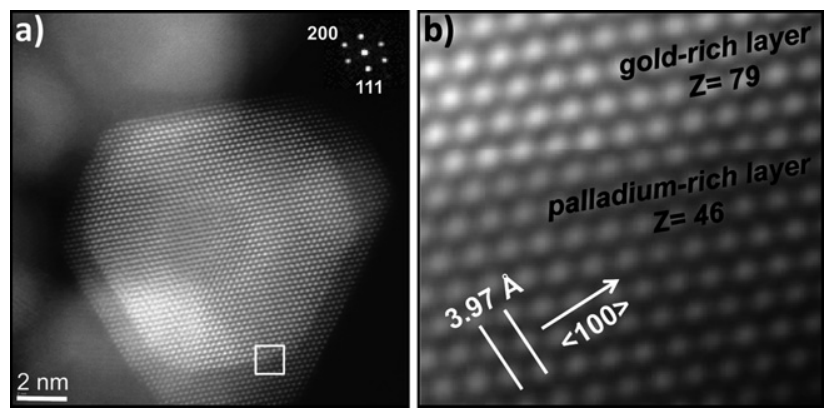

Fig. 2 Aberration-corrected STEM images. (a) Atomic-resolution HAADF-STEM image of a cuboctahedral Au/Pd nanoparticle. The contrast of the three distinct regions can be clearly seen. Bright dots represent atomic columns. The inset corresponds is the fast Fourier transform of the nanoparticle. (b) Enlarged image of a small part of the exterior layer of nanoparticle (indicated as a white square in (a)), exhibiting $<110>$ crystal orientation.

resolved. A detailed image of the outer shell is shown in Fig. 2b. The inset corresponds to the digital diffractogram of this image, which indicates that the nanoparticle is in the $<110>$ orientation. By measuring the distance between the individual atomic column peaks in the plot of intensity profile versus distance, an interplanar spacing was obtained, and the lattice parameter determined. The values obtained by the two methods were consistent, with a mean value of $3.97 \pm 0.08 \AA$ being obtained. This value is consistent with the one expected from Vegard's law, ${ }^{20}$ and indicates that despite the observed three-layer structure, which exhibits different composition domains, the lattice parameter is not significantly altered. Moreover, no 
extra diffraction spots, which would have suggested the presence of twinning or defects at the core-inner-shell and inner-shellouter-shell interfaces, were found. This strongly suggests that coherent interfaces are produced in these layered nanoparticles.

Another interesting feature of the particles studied is their single-crystal structure. We can simulate the HAADF contrast observed in the nanoparticles of Figs. 1 and 2 by assuming that the whole shape of the particle corresponds to a cuboctahedron. It is well established that projected atomic columns in HAADF-STEM images appear in bright contrast. Since the images are incoherent, no interference effects are produced between the Bloch waves propagating through the crystal. The contrast strongly depends on the atomic number. To a very good approximation, the detailed image is a convolution between a probe function and the projected atomic structure. We can approximate the image intensity as

$$
I=I_{\mathrm{p}} \otimes I\left(V_{\mathrm{p}}\right)
$$

where $I_{\mathrm{p}}$ is the probe effective intensity distribution and $I\left(V_{\mathrm{p}}\right)$ is the contribution of the projected potential. Now if we assume that two kinds of atoms are present, in any column of atoms, we can write:

$$
I\left(V_{\mathrm{p}}\right)=F\left(\mathrm{NAu}_{i}+\mathrm{NPd}_{j}\right) \times I(Z)
$$

where $F\left(\mathrm{NAu}_{i}+\mathrm{NPd}_{j}\right)$ corresponds to the atomic distribution of atoms in the particle, and $I(Z)$ is the intensity that originates from thermal diffuse scattering (TDS). In order to determine
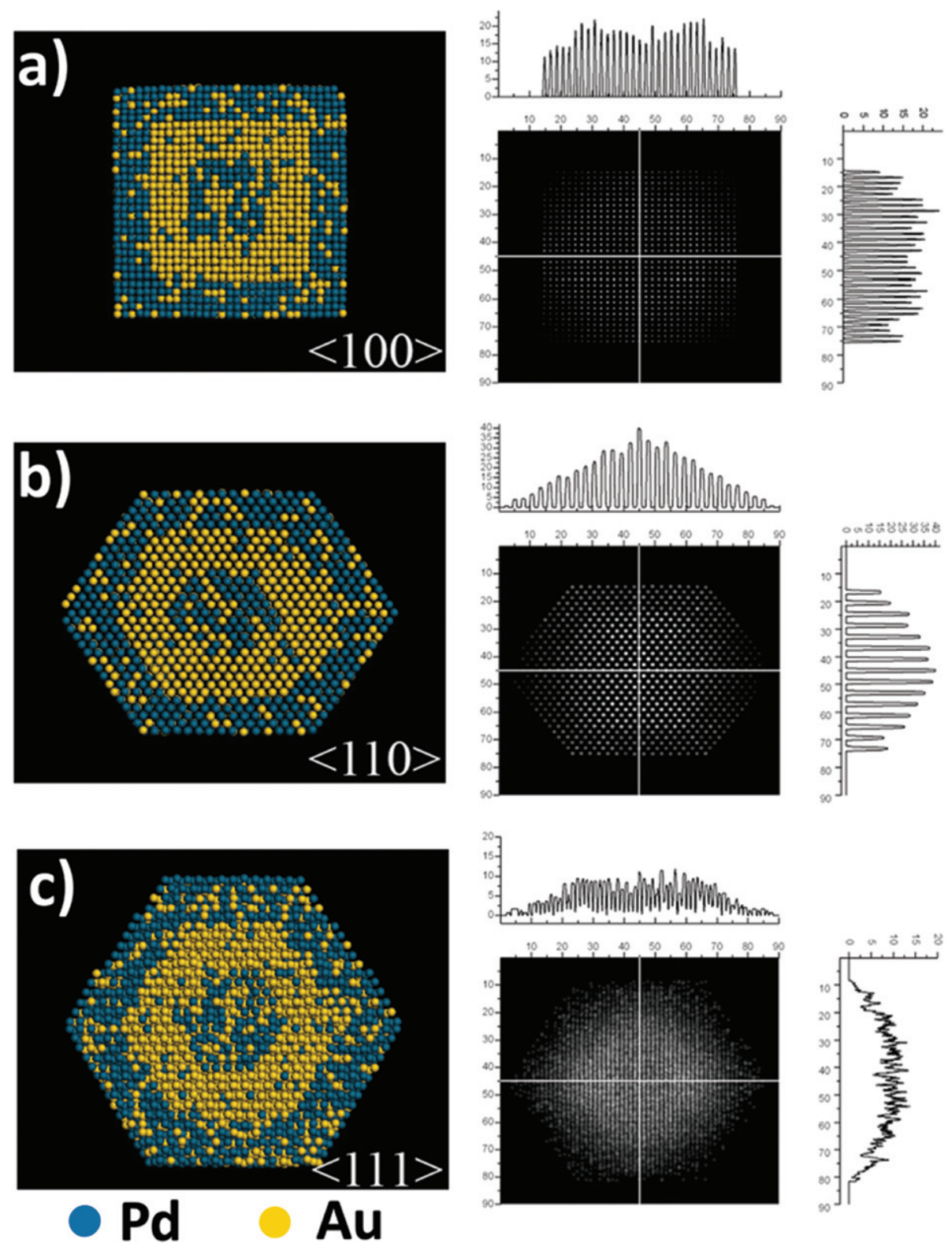

Fig. 3 Simulated HAADF-STEM of a cuboctahedral particle consisting of three layers: a Pd-rich inner-core with a thickness of five layers, an Au-rich intermediate-shell with a thickness of five layers, and a Pd-rich exterior-shell with a thickness of five layers. The cross-sectional view of Au/Pd models, their corresponding STEM images, and intensity profiles are shown for the (a) $<100>$, (b) $<110>$, and (c) $<111>$ orientations, respectively. 
$F\left(\mathrm{NAu}_{i}+\mathrm{NPd}_{j}\right)$, we assume a cuboctahedral-shaped particle formed of three layers: a central core containing $60 \%$ palladium, an intermediate shell containing $90 \%$ gold and an exterior shell containing $75 \%$ palladium. The $I(Z)$ was calculated assuming that the main contribution corresponded to TDS. ${ }^{21}$ The results for three different zone axes are shown in Fig. 3a-c. The displayed orientations, namely $<100>,<110>$ and $<111>$, exhibit a good agreement between theoretical and experimental images, in all cases.

A measurement of the theoretical inter-atomic column spacing was also consistent with the experimental measurements. However, a systematic study of the aberration-corrected HAADF-STEM images shows deviations from the ideal shape. For instance, in several cases the central core is more rounded than in the simulated case. When we compared an experimental HAADF intensity profile in a picture without atomic resolution
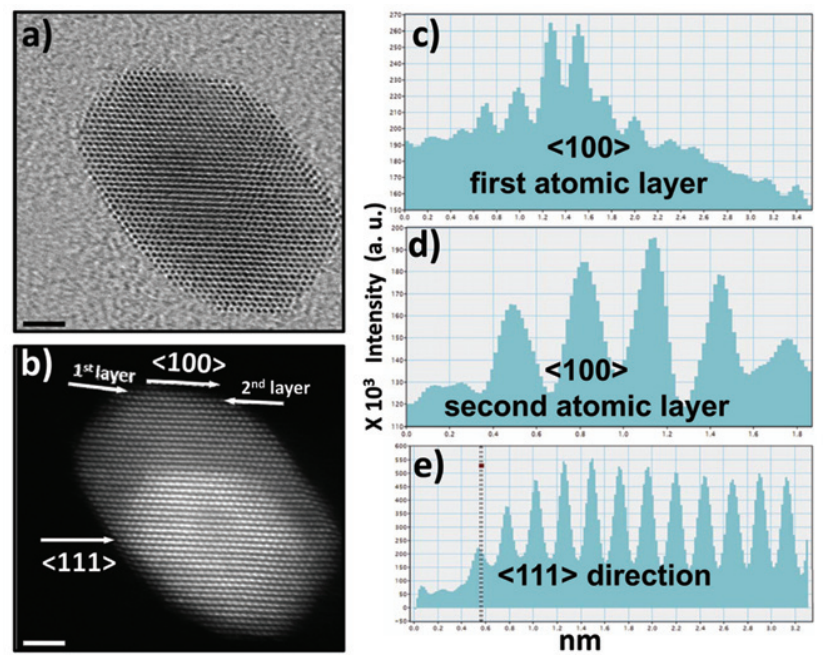

Fig. 4 Image of $\mathrm{Au} / \mathrm{Pd}$ nanoparticle showing deviation from ideal cuboctahedral morphology and its corresponding intensity profile along different crystal orientations. By employing aberration-corrected HRTEM under (a) bright field and (b) HAADF-STEM modes, the atomic positions and chemical species can be clearly analyzed. The scale bar corresponds to $2 \mathrm{~nm}$ in both cases. The intensity profiles for (c) the first and (d) the second atomic layers of the bimetallic nanoparticle along the $<100>$ surface and (e) the $<111>$ face show a Gaussian distribution.
(Fig. 1a,b) we found a profile that agrees quite well with the theoretical profile for a $<110>$-oriented cuboctahedral shape. The profile is consistent with a three-layer particle with a Pd-rich central core. No twins or stacking faults are seen on the interface between layers, indicating that the interfaces between layers are fully coherent along the axis. However, more detailed information can be obtained from aberration-corrected images. Another cuboctahedral particle is shown in Fig. 4a,b in STEM brightfield $(\mathrm{BF})$ mode and HAADF mode, respectively. The fast Fourier transform (FFT) of both images can be readily indexed as corresponding to the $\langle 110\rangle$ zone axis. Since the HAADFSTEM intensity is sensitive to the atomic number and to the mass thickness, we can obtain profiles of different rows of atomic columns. The results are shown in Fig. 4c-e for the first and second atomic rows of the $<100>$ surface and the $<111>$ atomic row. The measurements were taken along the directions indicated by the arrows shown in Fig. 4b. As can be seen, the profiles show an increase in intensity and then a decrease, displaying a Gaussian distribution.

A close examination of the regular cuboctahedral structure shown in Fig. 5 indicates that the intensity in the $<100>$ direction should always be increasing (Fig. 5a), and that the profile in the $<111>$ direction should display constant intensity. The only possibility to reconcile the experimental data with the $\mathrm{Au} / \mathrm{Pd}$ three-layer model (Fig. 5b) is that two phenomena are occurring. First, the particle is not smooth, and contains edges, steps and kinks (Fig. 5c), and secondly there is atomic mixing of $\mathrm{Au}$ and $\mathrm{Pd}$ in the columns on or near the surface. From consideration of the theoretical models included in this report, we conclude that the addition of a second metal introduces defects to the surface of the particles. The lack of extra diffraction spots in the electron diffraction patterns of the particles (Fig. 1c) indicates that these are single crystals. The absence of defects in the electron diffraction patterns is an intriguing fact that requires additional experimental analysis.

\section{Conclusions}

In summary, this paper reports the direct observation of individual atomic columns of nanoparticles with three-layer morphology. This enabled us to investigate their structurecomposition relations. A very important principle for bimetallic
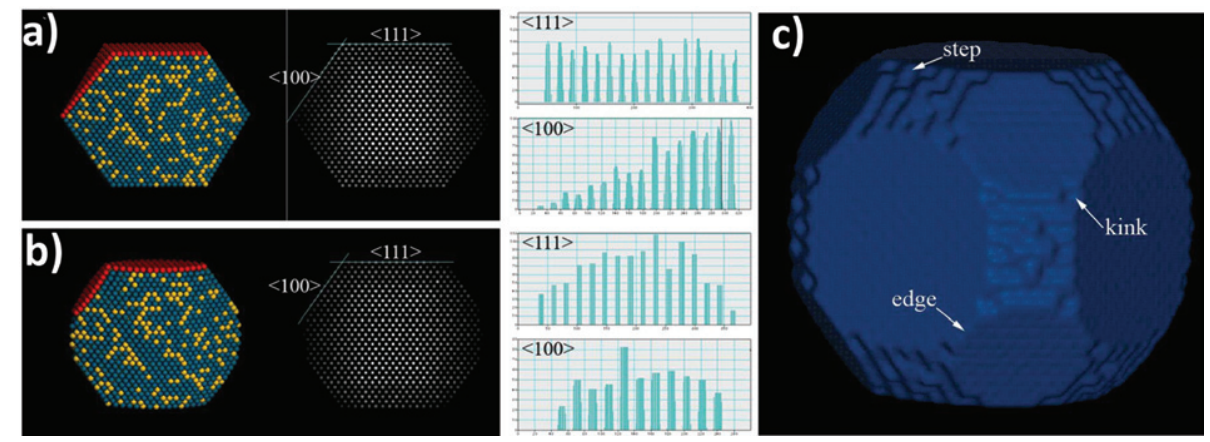

Fig. 5 Models corresponding to (a) a regular cuboctahedron and (b) Au/Pd modified cuboctahedron. The thickness of the atomic columns increases for $\mathrm{a}<100>$ face, whereas is constant for a $<111>$ face for the regular cuboctahedron. The model of the reconstructed shape of Au/Pd nanoparticles is consistent with the observed intensity profiles. (c) Solid polyhedron representing an Au/Pd nanoparticle, showing steps, edges and kinks on the surface. Locally, these features produce high-index surfaces. 
particles was elucidated, namely that for nanoparticles displaying alloying and a core-shell structure, differences in atomic radii and atom mobilities causes the formation of high Miller index facets. However, these facets are unstable and decompose into steps formed composed of $\{100\}$ and $\{111\}$ facets. It is very likely that the stepped surface of analyzed nanoparticles plays an important role in the catalytic activity of bimetallic systems.

\section{Acknowledgements}

The authors thank the Center for Nano and Molecular Science and Technology at the Texas Materials Institute, and the International Center for Nanotechnology and Advanced Materials at The University of Texas at Austin. We also thank the support from the Welch Foundation Grant F1615, and the NSF Grant DMR-0602587: "Alloys at the Nanoscale: The case of Nanoparticles". Electron microscopy at Oak Ridge National Laboratory's High Temperature Materials Laboratory was sponsored by the Assistant Secretary for Energy Efficiency and Renewable Energy, Office of FreedomCAR and Vehicle Technologies, as part of HTML's National User Program, ORNL, managed by UT-Battelle LLC, for the U.S. Department of Energy under contract number DE-AC05-00OR22725.

\section{References}

1 N. Dellby, O. L. Krivanek, P. D. Nellist, P. E. Batson and A. R. Lupini, J. Electron Microsc., 2001, 50, 177.

2 Y. Peng, P. D. Nellist and S. J. Pennycook, J. Electron Microsc., 2004, 53, 257.
3 J. L. Hutchison, J. M. Titchmarsh, D. J. H. Cockayne, R. C. Doole, C. J. D. Hetherington, A. I. Kirkland and H. Sawada, Ultramicroscopy, 2005, 103, 7.

4 E. C. Cosgriff and P. D. Nellist, J. Electron Microsc,, 2001, 50, 291.

5 K. Ishizuka, J. Electron Microsc., 2004, 53, 129.

6 J. Yamasaki, T. Kawai and N. Tanaka, Microsc. Microanal., 2006, 12, 285.

7 A. M. Sanchez, Microsc. Microanal., 2006, 12, 483.

8 D. A. Blom, L. F. Allard, S. Mishina and M. A. O'Keefe, Microsc. Microanal., 2006, 12, 483.

9 C. Noguez, J. Phys. Chem. B, 2007, 111, 3806.

10 S. J. Mejía-Rosales, C. Fernández-Navarro, E. Pérez-Tijerina, D. A. Blom, L. F. Allard and M. José-Yacamán, J. Phys. Chem. C, 2007, 111, 1256.

11 H. Nitani, J. Nanopart. Res., 2006, 8, 951.

12 A. Steinbrueck, A. Csaki, G. Festag and W. Fritzsche, Plasmonics, 2006, 1, 79.

13 E. Pérez-Tijerina, M. Gracia Pinilla, S. Mejía-Rosales, U. OrtizMéndez, A. Torres and M. José-Yacamán, Faraday Discuss., 2008, 138, 353.

14 G. Kickelbick and L. M. Liz-Marzan, 'Core-shell nanoparticles', in: Encyclopedia of Nanoscience and Nanotechnology, American Scientific Publishers, Valencia, California, USA, 2004, vol. 2, p. 199.

15 L. M. Liz-Marzan, Mater. Today, 2004, 7, 26.

16 D. Ferrer, A. Torres-Castro, X. Gao, S. Sepúlveda-Guzmán, U. Ortiz-Méndez and M. José-Yacamán, Nano Lett., 2007, 7, 1701.

17 The SimulaTem software can be obtained from Dr A. Gomez at the Instituto de Física, UNAM, México, D.F.

18 K. Ishizuka, Ultramicroscopy, 2001, 90, 71

19 K. Andrews, D. J. Dyson and S. R. Keown, Interpretation of electron diffraction patterns. Plenum Press, New York, 1971.

20 A. R. Denton and N. W. Ashcroft, Phys. Rev. A, 1991, 43, 3161.

21 M. D. Croitoru, D. Van Dyck, S. V. Aert, S. Bals and J. Verbeeck, Ultramicroscopy, 2006, 106, 933. 